Purdue University Purdue e-Pubs

$6-2013$

\title{
The Use of Equivalent Source Models for Reduced Order Simulation in Room Acoustics
}

\author{
Yangfan Liu \\ Purdue University, liu278@purdue.edu \\ J Stuart Bolton \\ Purdue University, bolton@purdue.edu
}

Follow this and additional works at: http:/ / docs.lib.purdue.edu/herrick

Liu, Yangfan and Bolton, J Stuart, "The Use of Equivalent Source Models for Reduced Order Simulation in Room Acoustics" (2013). Publications of the Ray W. Herrick Laboratories. Paper 80.

http://docs.lib.purdue.edu/herrick/80

This document has been made available through Purdue e-Pubs, a service of the Purdue University Libraries. Please contact epubs@purdue.edu for additional information. 


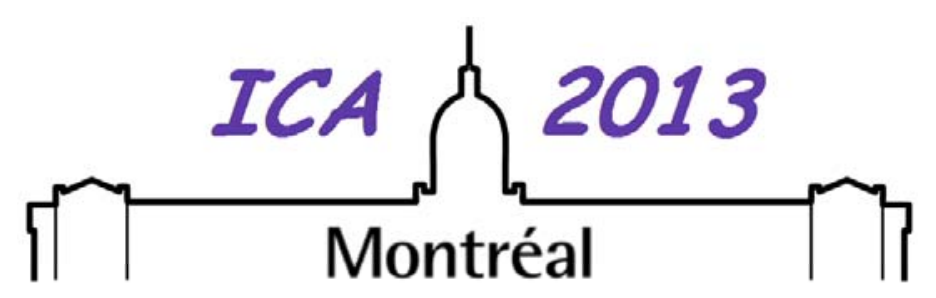

The Use of Equivalent Source Models for Reduced Order Simulation in Room Acoustics

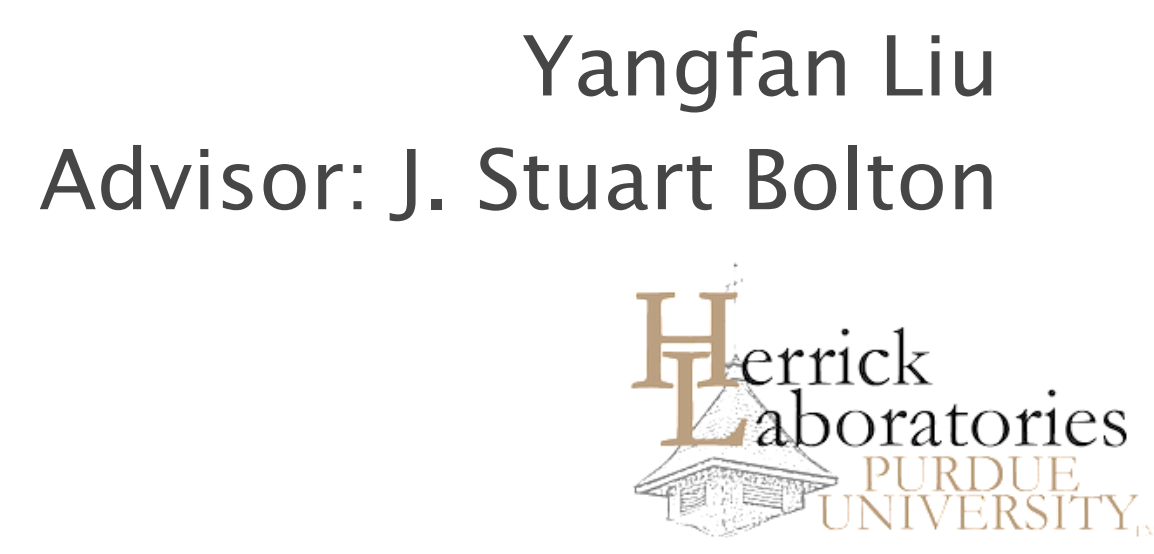




\section{Motivation}

\section{$\square$ Room Acoustics with Source of Finite Size}

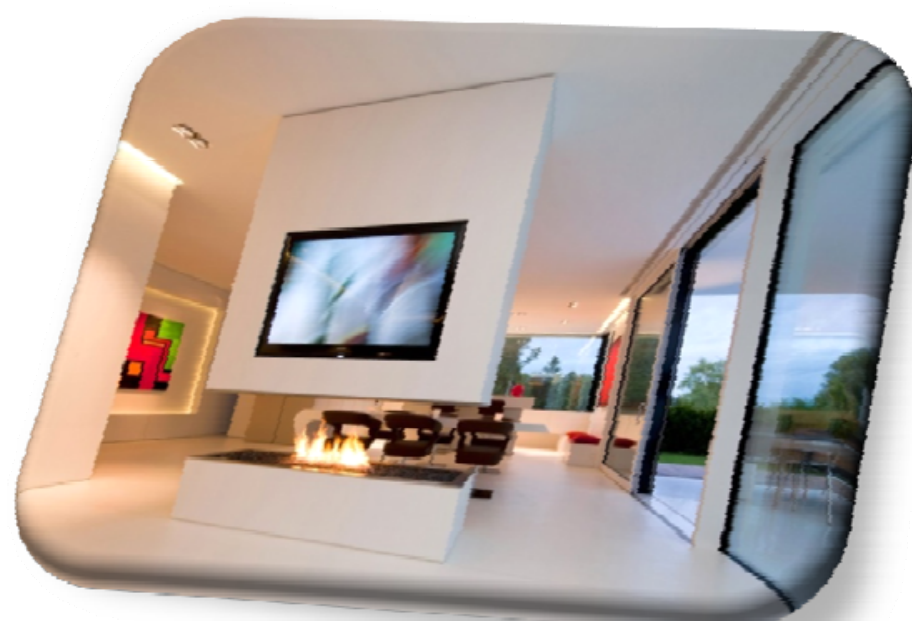

Available techniques are not suitable for a fast and accurate room acoustics simulations of finite-size sources

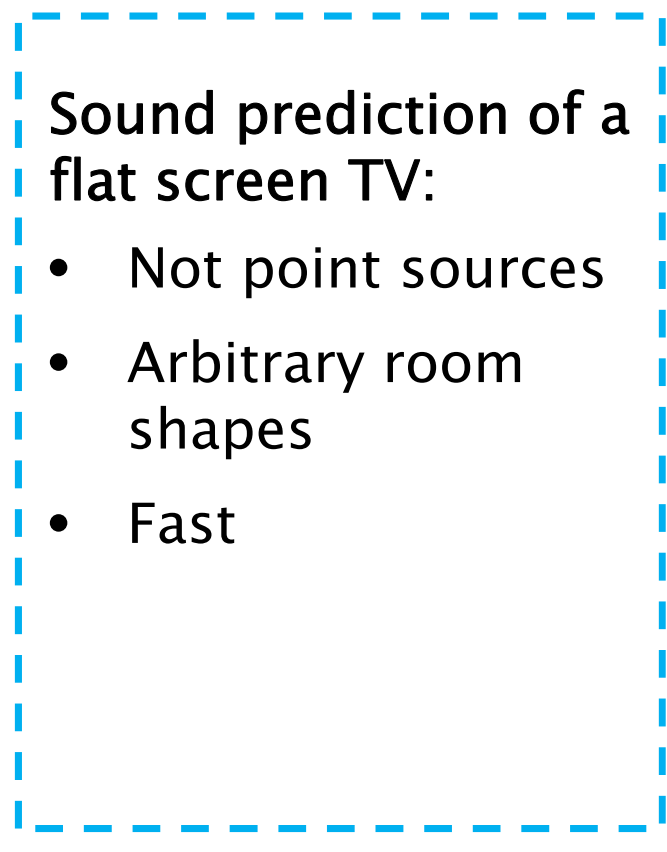

Therrick

aboratories a PURDLE

\section{$\square$ The Use of Equivalent Source Models (ESM)}

ESM in General:

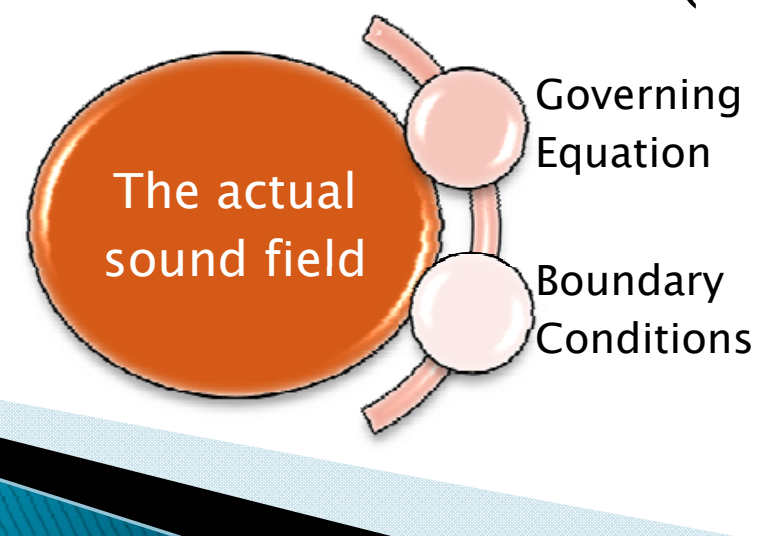

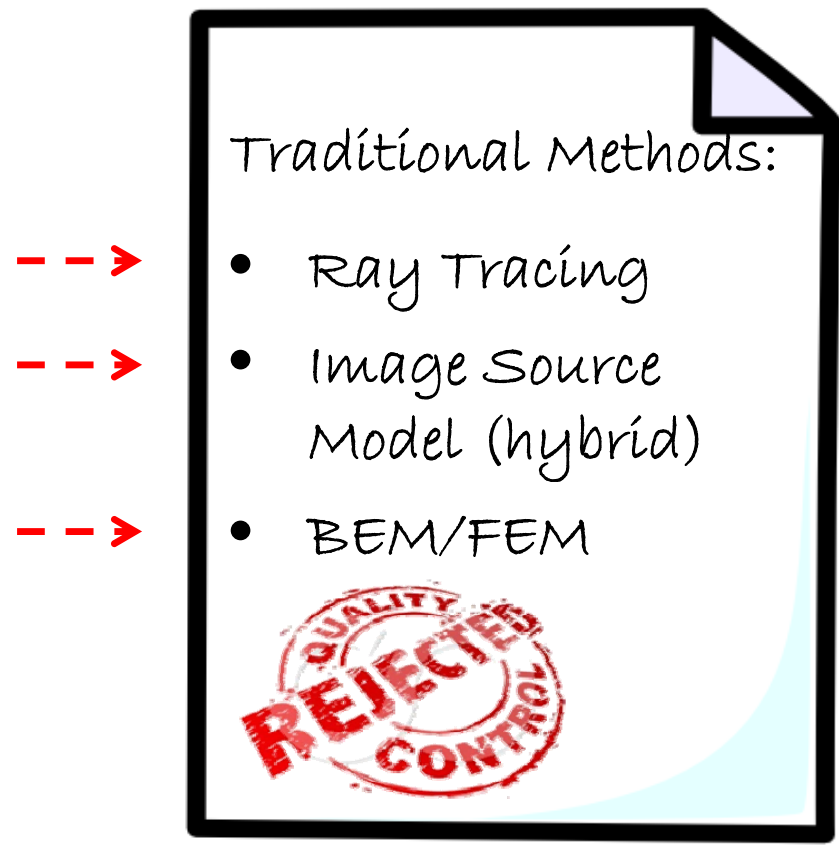

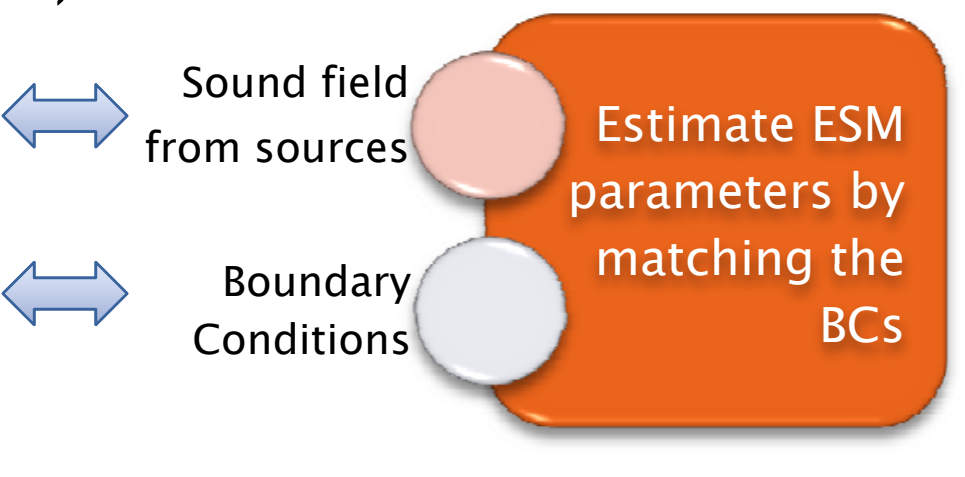




\section{ESM in Room Acoustics}

Sound Field Components in Room Acoustics

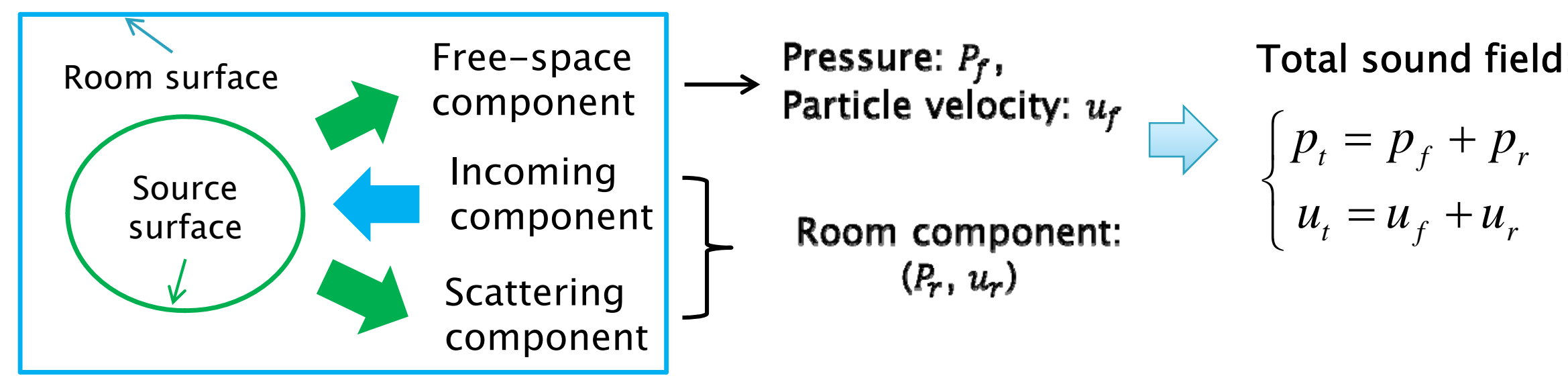

$\square$ Free-space ESM vs. Room Acoustics ESM

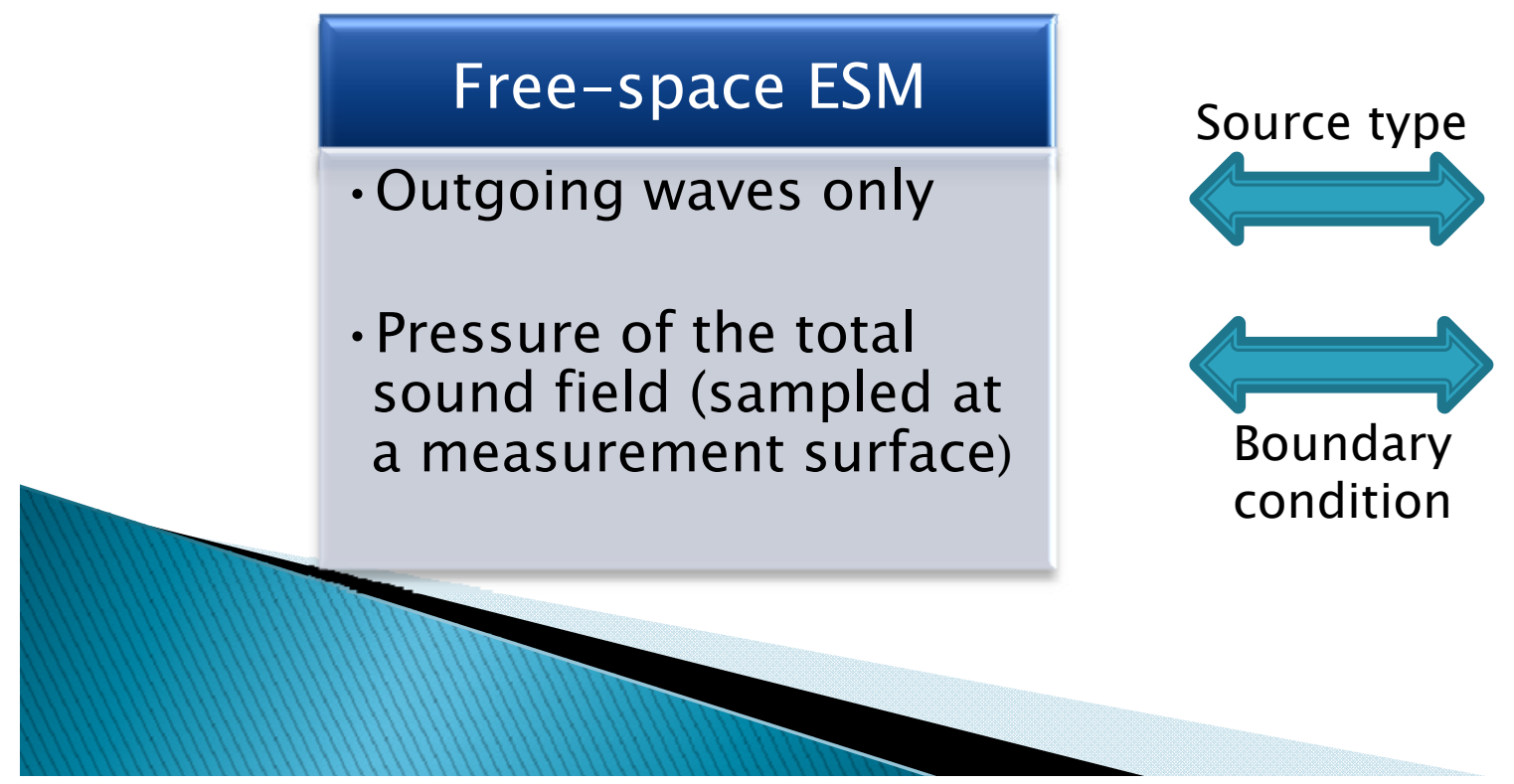

\begin{tabular}{|l|}
\hline Room Acoustics ESM \\
\hline - Both incoming and \\
outgoing waves \\
- Impedances of room \\
component (sampled at \\
both source and room \\
surfaces)
\end{tabular}




\section{Boundary Conditions}

$\square \mathrm{BC}$ of the Room Components in Room Acoustics

- BC on source surface, $\Gamma_{1}$, (admittance $\beta_{1}$ ): Total: Free-space: $\quad \beta_{1}(x) p_{f}(x)+u_{0}(x)=u_{n f}(x)$ $\ltimes$

In-vacuo driving velocity on the source surface. (source characteristics)

- $B C$ on room surface, $\Gamma_{2}$, (admittance $\left.\beta_{2}\right)$ :

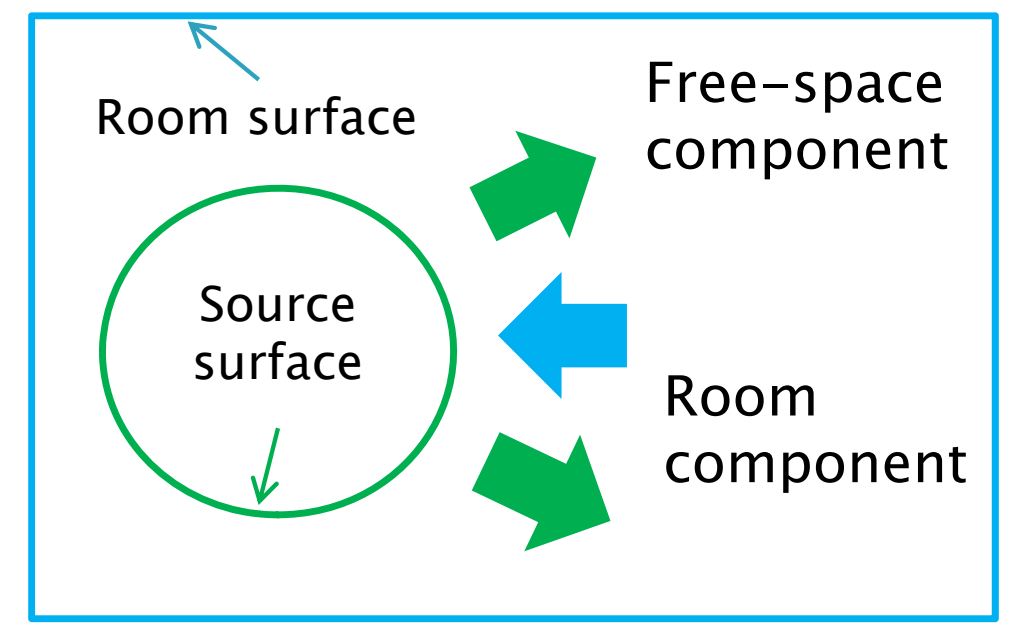

Total: $\quad \beta_{2}(x) p_{t}(x)=u_{n t}(x)$

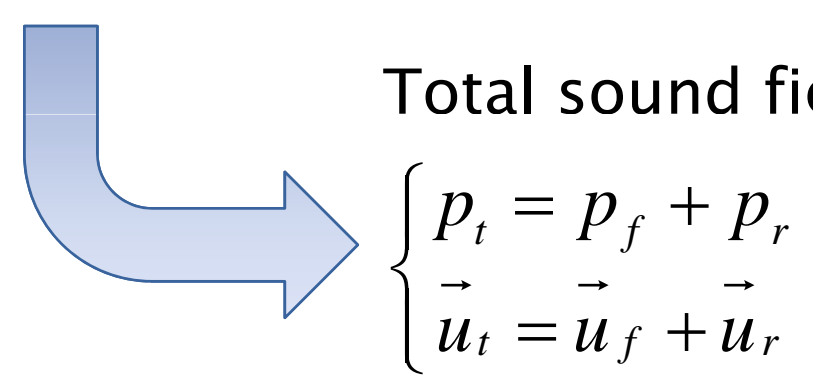

Boundary conditions used in the room acoustics ESM:

$$
\left\{\begin{array}{l}
p_{t}=p_{f}+p_{r} \\
\vec{u}_{t}=\vec{u}_{f}+\vec{u}_{r}
\end{array}\right.
$$$$
\left\{\begin{array}{cc}
\beta_{1}(x) p_{r}(x)-u_{n r}(x)=0 & x \in \Gamma_{1} \\
\beta_{2}(x) p_{r}(x)-u_{n r}(x)=u_{n f}(x)-\beta_{2}(x) p_{f}(x) & x \in \Gamma_{2}
\end{array} .\right.
$$ 


\section{Construction of ESM in General}

General Procedure of Constructing Room Acoustics ESM

After choose the type
of equivalent sources: \{

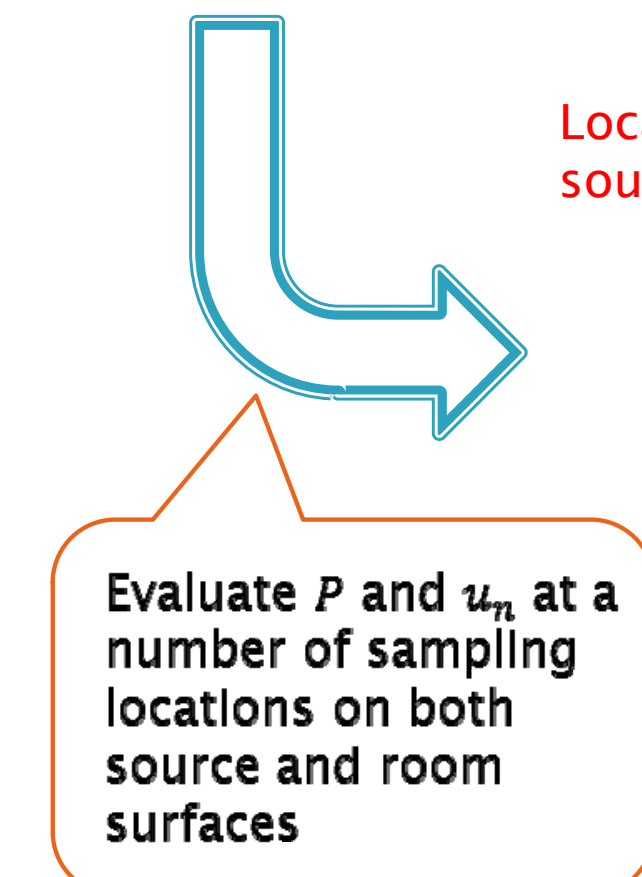

Location in the sound field
- $g_{t}\left(x, y_{t}\right)$ - sound field of source with unit strength

- $Q_{t}$ - the source strength

Location of each source

Matrix form used to estimate the ESM parameters:

$$
\left[\begin{array}{c}
B_{1} A_{p}^{(1)}-A_{u_{n}}^{(1)} \\
B_{2} A_{p}^{(2)}-A_{u_{n}}^{(2)}
\end{array}\right] \vec{Q}=\left[\begin{array}{c}
0 \\
\vec{u}_{n f}-B_{2} \vec{p}_{f}
\end{array}\right]-\rightarrow \begin{aligned}
& \text { Boundary condition } \\
& \text { on source surfaces } \\
& \text { on roundary condition }
\end{aligned}
$$

$\left[B_{1}=\operatorname{diag}\left(\beta_{1}\left(x_{1}\right), \beta_{1}\left(x_{2}\right), \ldots, \beta_{1}\left(x_{M_{1}}\right)\right)\right.$, $B_{2}=\operatorname{diag}\left(\beta_{2}\left(x_{M_{1}+1}\right), \beta_{2}\left(x_{M_{1}+2}\right), \ldots, \beta_{2}\left(x_{M}\right)\right)$ $\left(A_{p}^{(1)}\right)_{i j}=g_{j}\left(x_{i}, y_{j}\right), \quad\left(A_{u_{n}}^{(1)}\right)_{i j}=\frac{1}{j \omega \rho_{0}} \partial_{n} g_{j}\left(x_{i}, y_{j}\right)$, $\left(A_{p}^{(2)}\right)_{i j}=g_{j}\left(x_{M_{1}+i}, y_{j}\right), \quad\left(A_{u_{n}}^{(2)}\right)_{i j}=\frac{j \omega \rho_{0}}{j \omega \rho_{0}} \partial_{n} g_{j}\left(x_{M_{1}+i}, y_{j}\right)$,

Construct a specific ESM L (find the sound field expression) 


\section{Two Types of ESMs}

$\square$ Room Acoustics ESM Using Monopole Distributions

- Monopoles are distributed inside the source surface (outgoing wave) and outside the room surface (incoming wave)

- Sound field expression (2D):

$$
g(x, y)=\frac{j}{4} H_{0}^{(1)}(k r)
$$

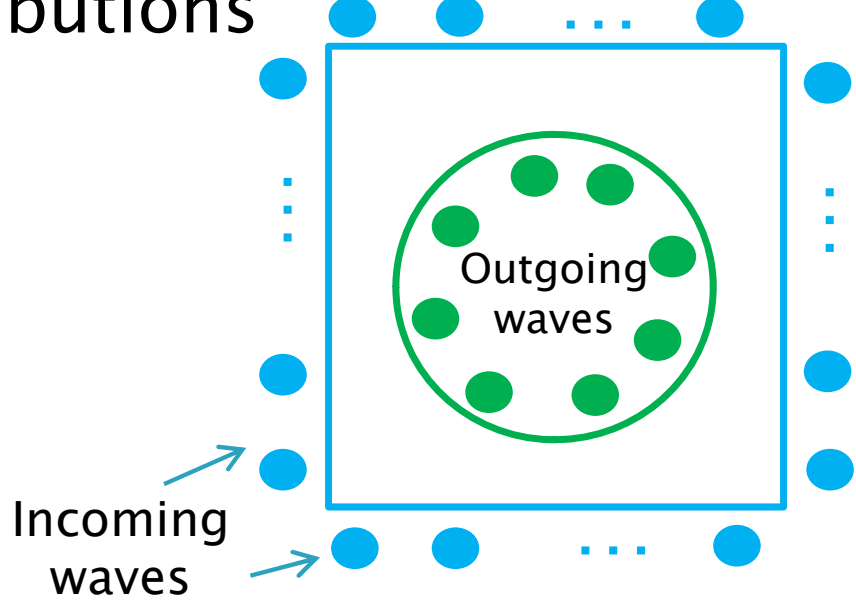

$\square$ Room Acoustics ESM Using Monopole Distributions

- Multipoles of monopole, dipole, quadrupole, ... $n$th order, ... (both incoming and outgoing)

- Sound field expression (2D):

$$
n=0: \quad P_{0}^{\text {out }}(x, y)=\frac{j}{4} H_{0}^{(1)}(k r), \quad P_{0}^{\text {in }}(x, y)=\frac{j}{4} H_{0}^{(2)}(k r)
$$

$n$th order:

$$
\left\{\begin{array}{c}
P_{S n}^{\text {out }}=S_{n} P_{n}^{\text {out }}=S_{n} R_{n}\left(P_{0}^{\text {out }}\right) \cdot \vec{v}_{1} \cdot \vec{v}_{2} \ldots \cdot \vec{v}_{n} \\
P_{S n}^{\text {in }}=S_{n} P_{n}^{\text {in }}=S_{n} R_{n}\left(P_{0}^{\text {in }}\right) \cdot \vec{v}_{1} \cdot \vec{v}_{2} \ldots \cdot \vec{v}_{n}
\end{array}\right.
$$

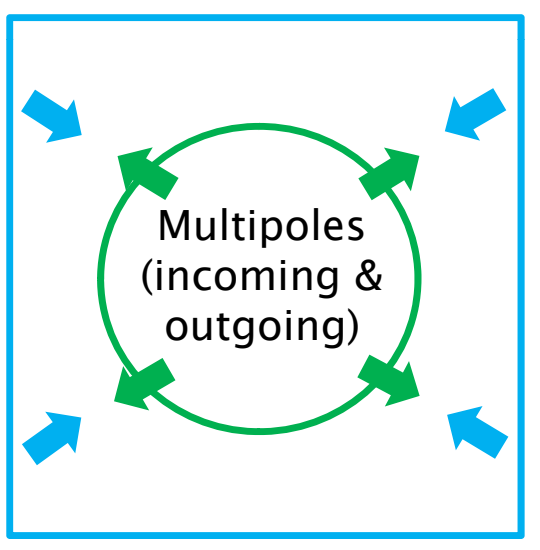

$R_{n}=\nabla^{\otimes n}$

$\otimes$ - tensor outer product - tensor inner product 


\section{Sound field of Multipole Sources}

\section{aSound field expression for multipole sources (2D):}

Sound field of order $n+1$ source and order $n$ source can be related by directional derivative:

$$
\begin{aligned}
P_{S n+1}\left(\vec{X} \mid \overrightarrow{X_{0}}, \omega\right) & =d<\nabla P_{S n}\left(\vec{X} \mid \overrightarrow{X_{0}}, \omega\right), \vec{v}_{n+1}> \\
& =d<\left[\frac{\partial P_{S n}\left(\vec{X} \mid \overrightarrow{X_{0}}, \omega\right)}{\partial x_{0}}, \frac{\partial P_{S n}\left(\vec{X} \mid \overrightarrow{X_{0}}, \omega\right)}{\partial y_{0}}\right]^{T}, \vec{v}_{n+1}>
\end{aligned}
$$

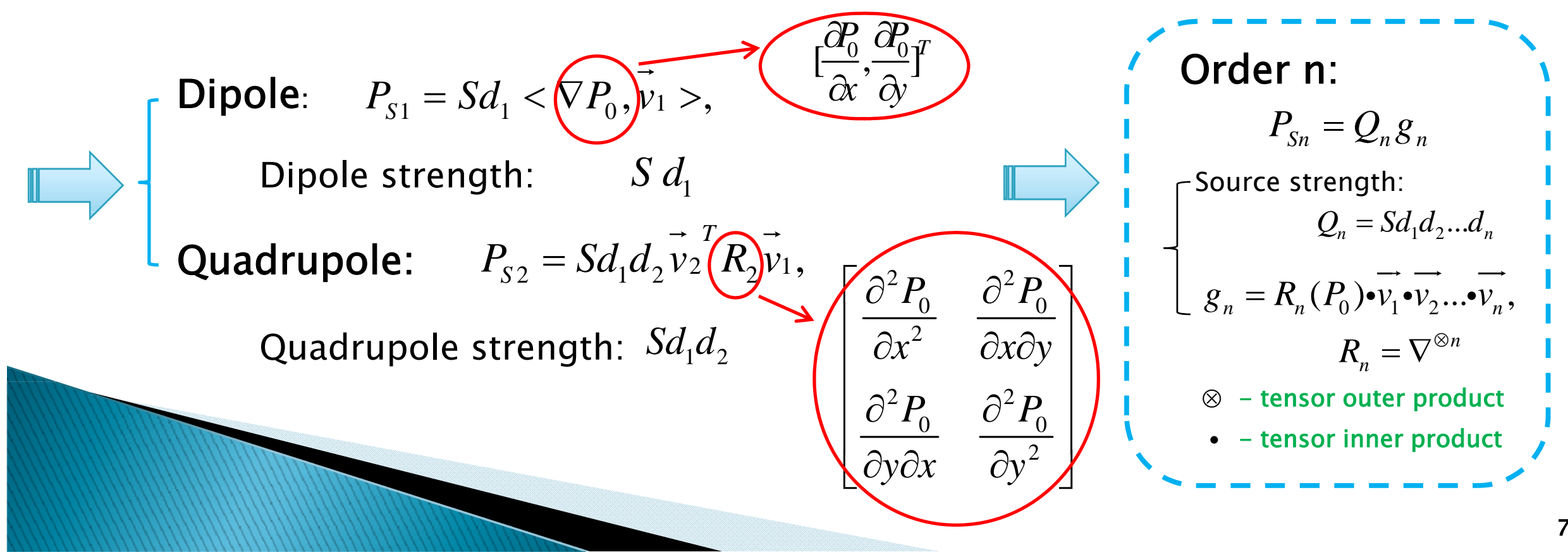




\section{Decomposition of the Multipoles}

For a source of order $n(n>1)$, there could be Infinitely many types because of the orientation vectors.

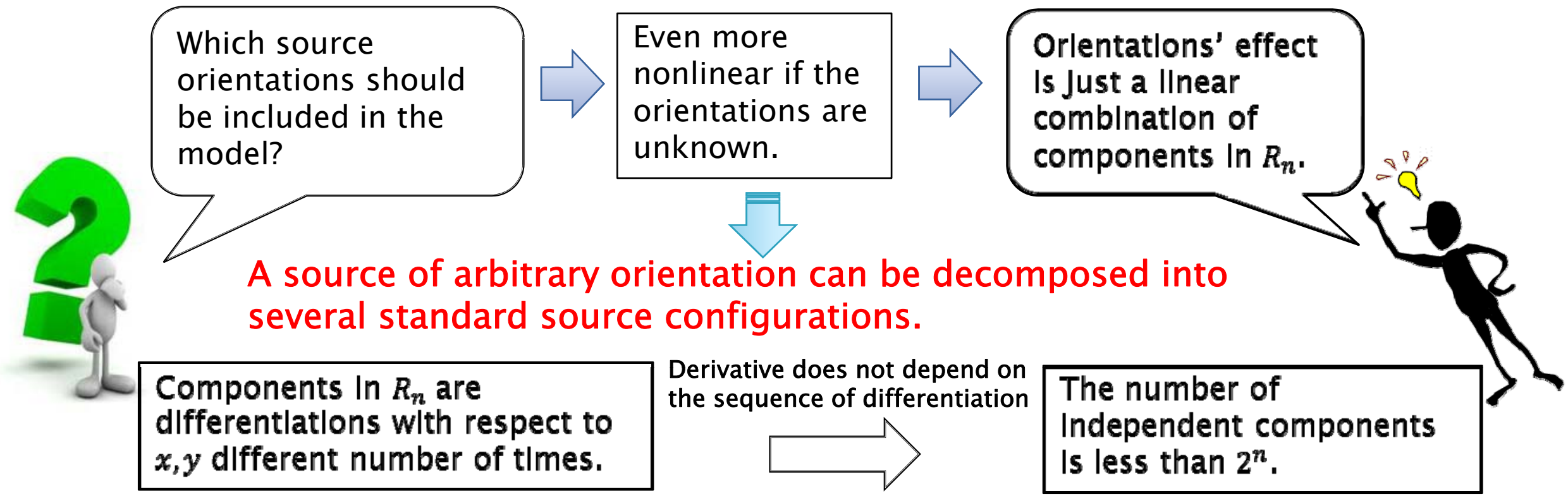

$\square$ How many independent elements?

$\begin{array}{ll}n \text {th order source, } & \\ \text { Iin } \mathbb{R}-\mathbb{D} \text { space: }\end{array}$
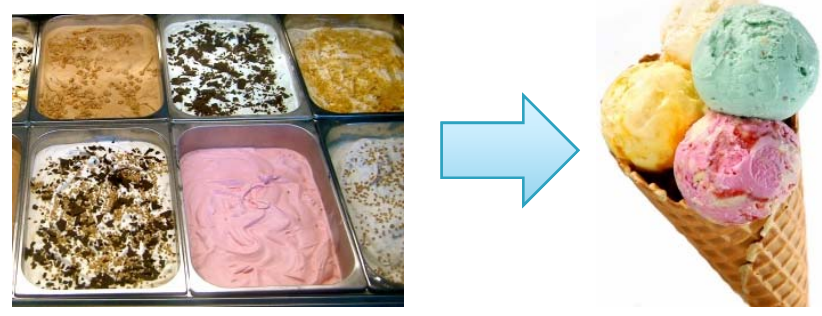

- Can be numerically enumerated for an arbitrary $n$. 


\section{Simulation Setup}

Simulation in a 2 dimensional room (circular geometry)

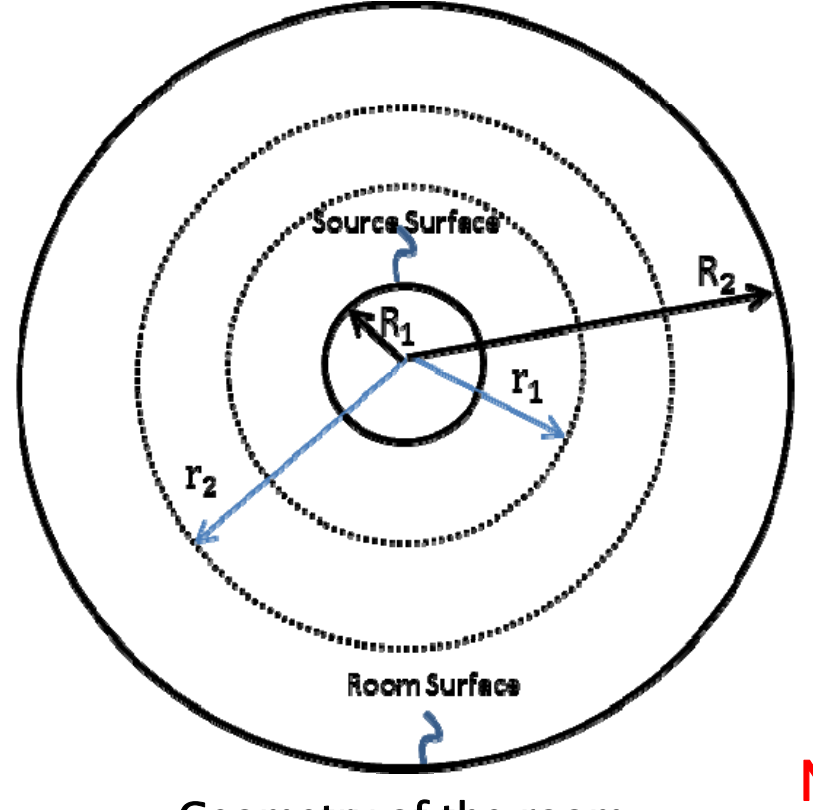

Geometry of the room

- $R_{1}=0.5 \mathrm{~m}$ (source surface),

- $R_{2}=2 \mathrm{~m}$ (room surface),

- $r_{1}=1 \mathrm{~m}$ (100 microphones),

- $r_{2}=1.5 \mathrm{~m}$ (100 microphones).
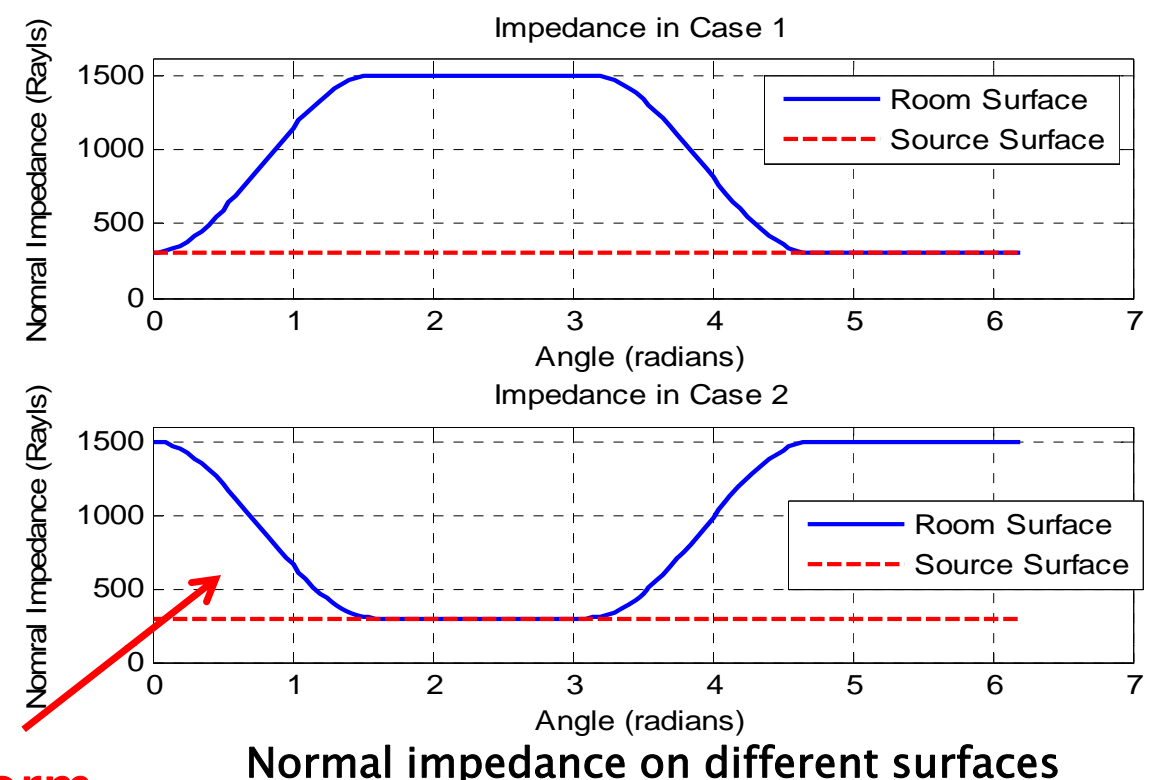

Non-uniform

Normal impedance on different surfaces

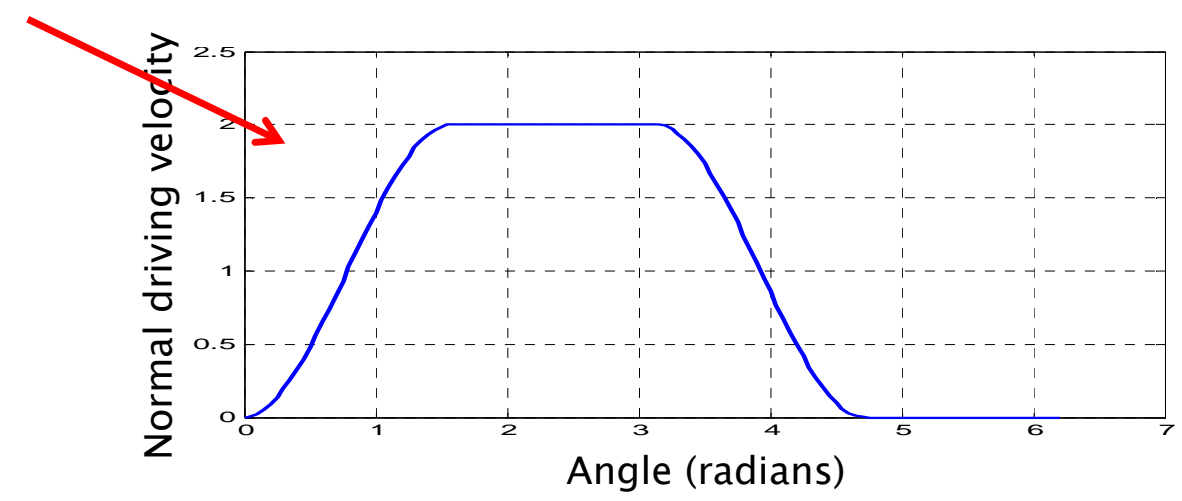

In-vacuo Driving velocity on the source surface 


\section{Models Used in Simulations}

\section{$\square$ Different models used in the simulation}

\begin{tabular}{cc}
\hline \hline Type of ESM & Number of Parameters \\
\hline Multipole ESM (order up to 3) & 10 \\
Multipole ESM (order up to 6) & 28 \\
Monopole ESM (outside: 100; inside: 30) & 130 \\
Monopole ESM (outside: 150; inside: 45) & 195 \\
Monopole ESM (outside: 200; inside: 60) & 260 \\
Monopole ESM (outside: 1000; inside: 300) & 1300 \\
\hline \hline
\end{tabular}

- Result from a Boundary Element Model was used as the true sound field.

\section{$\square$ Choice of regularization techniques}
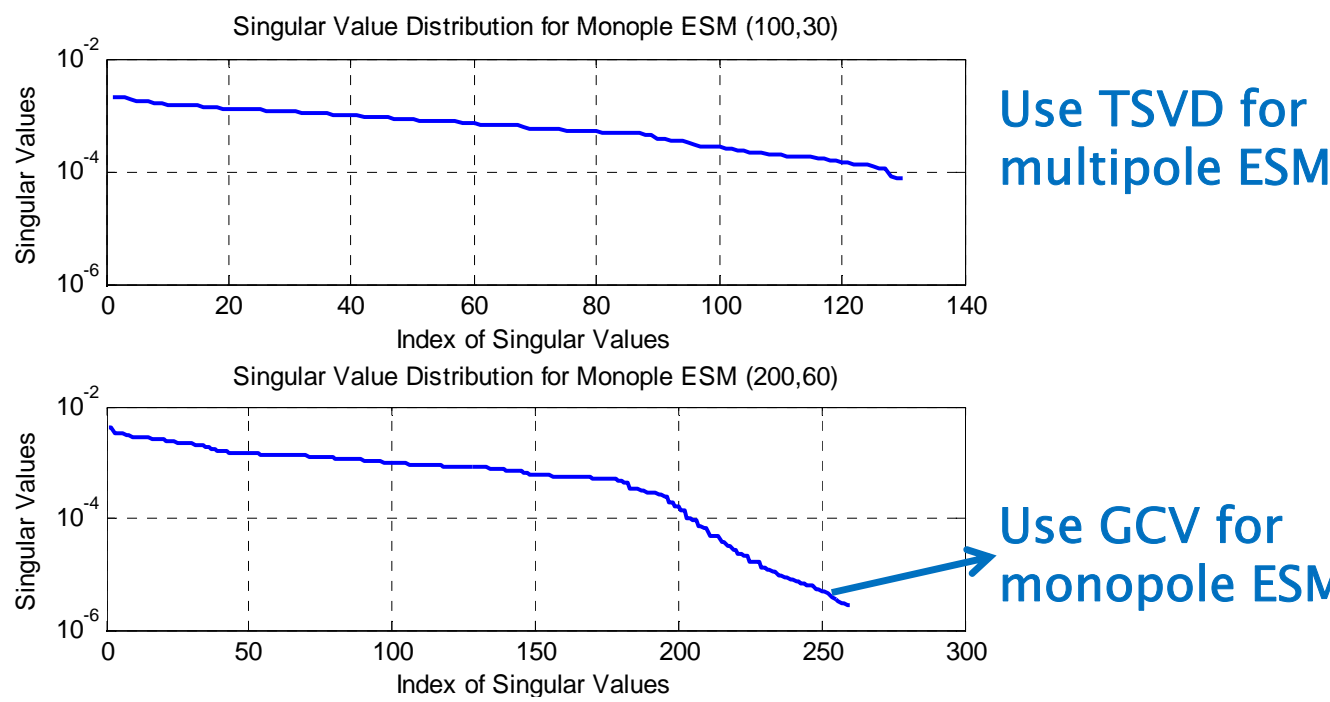

Use GCV for monopole ESM

Singular value distribution in monopole ESMs
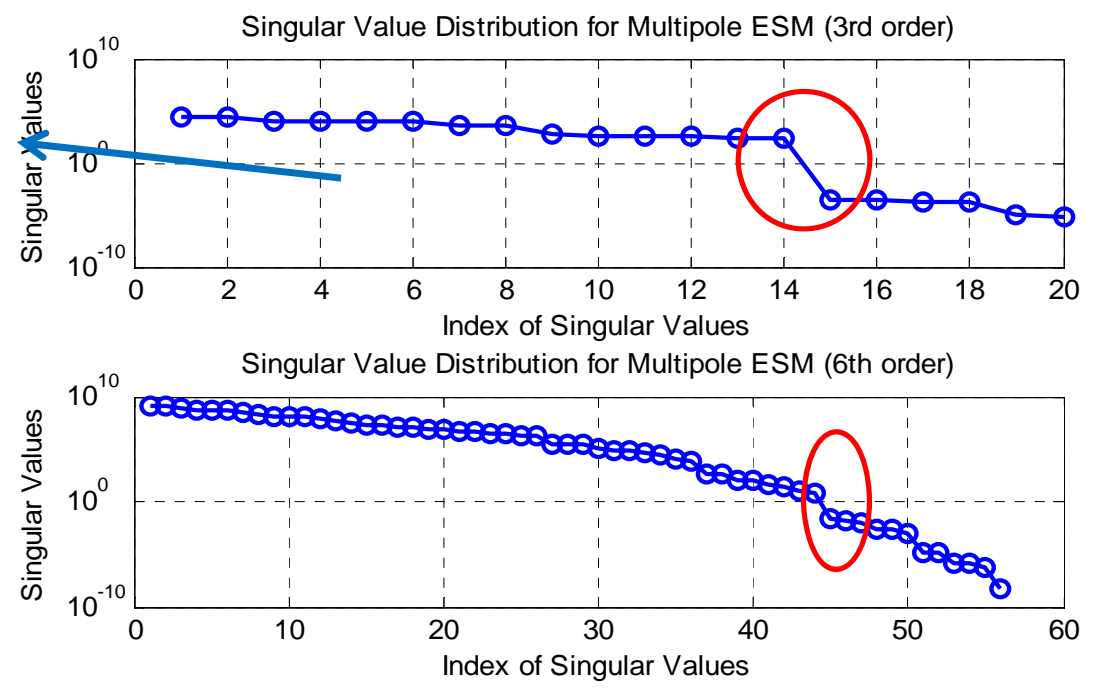

Singular value distribution in multipole ESMs 


\section{Analysis of Results}

\section{$\square$ Results from monopole distribution ESMs}
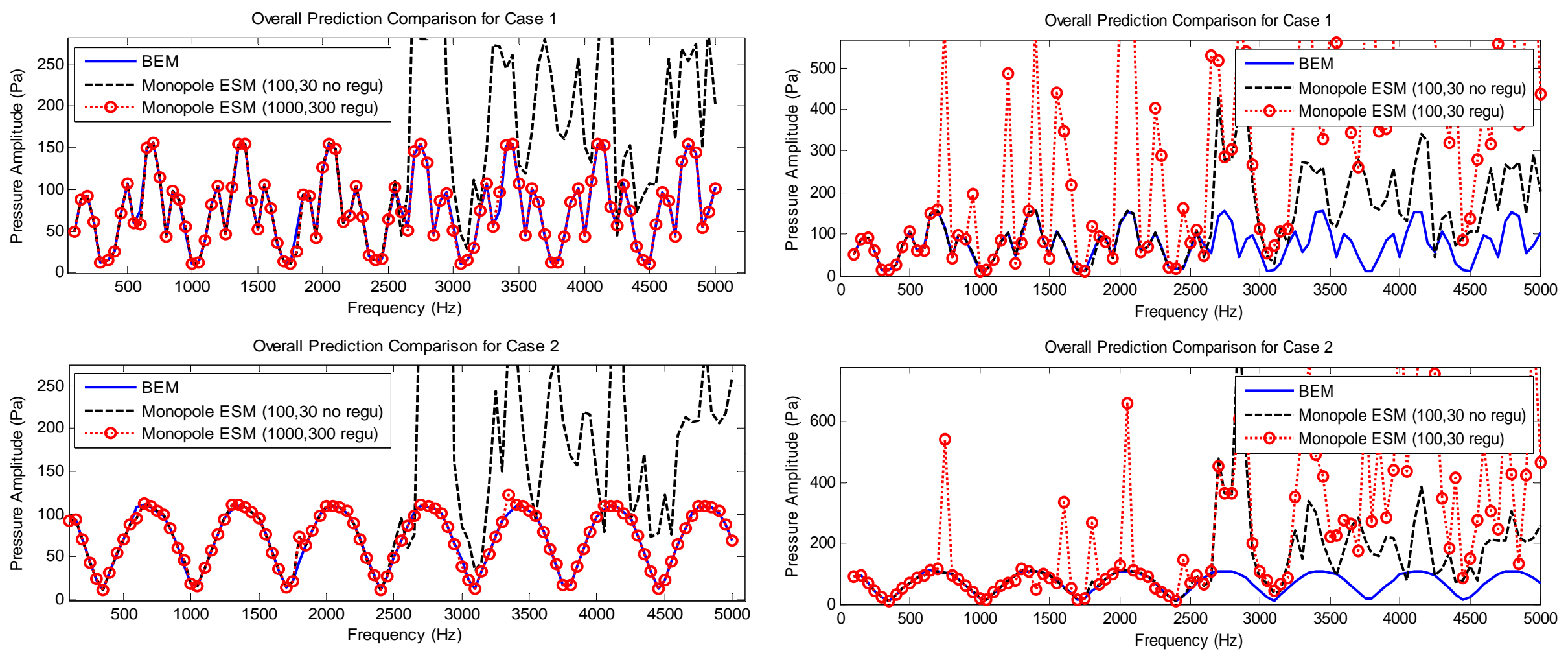

Spatially averaged prediction from monopole ESMs

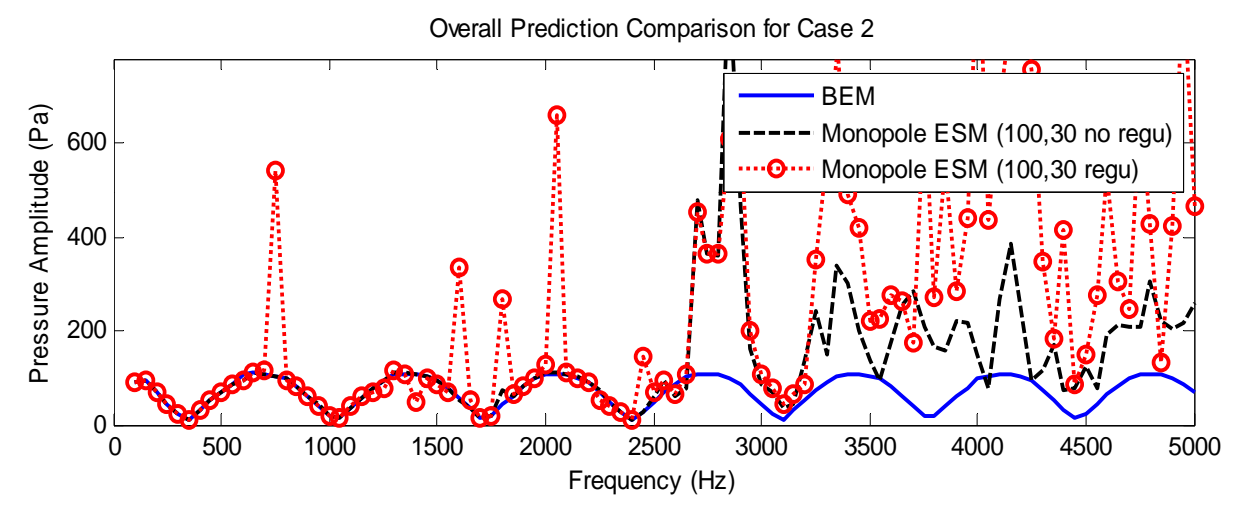

Spatially averaged prediction from monopole ESMs

- Accurate (up to $5000 \mathrm{~Hz}$ ) if there are a large number of monopoles and with regularization.

- Regularization may cause instabilities. 


\section{Analysis of Results}

\section{$\square$ Results from monopole distribution ESMs}
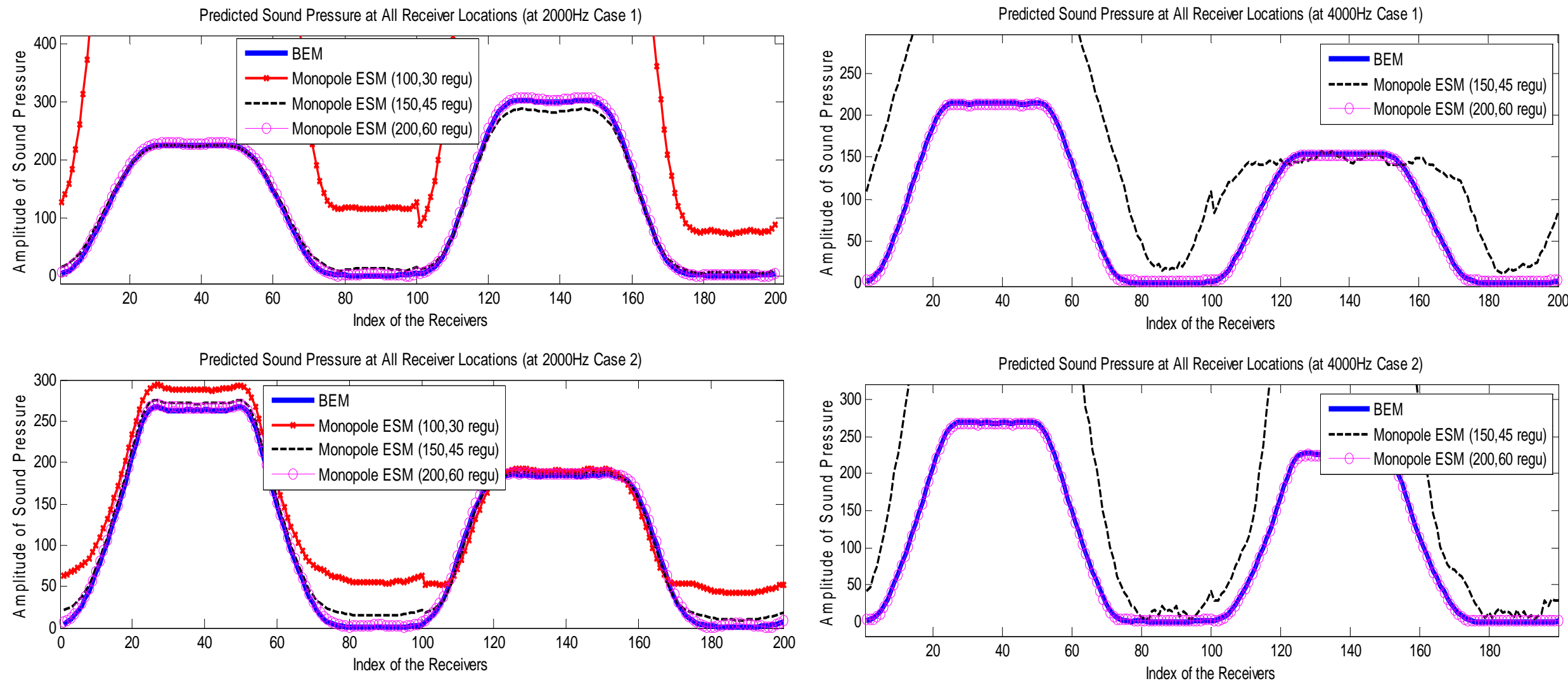

Prediction from monopole ESMs at $2000 \mathrm{~Hz}$

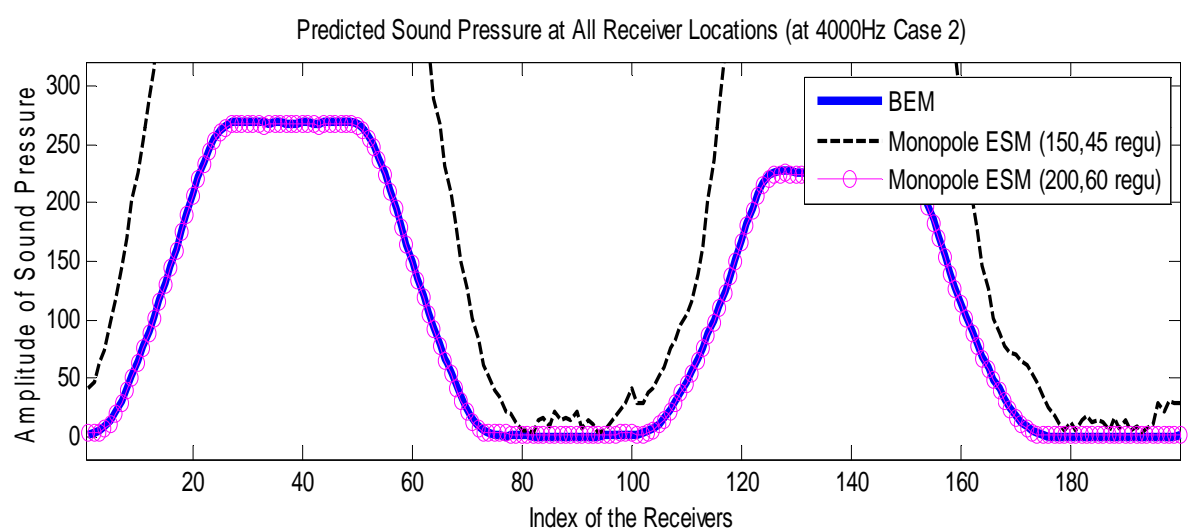

Prediction from monopole ESMs at $4000 \mathrm{~Hz}$

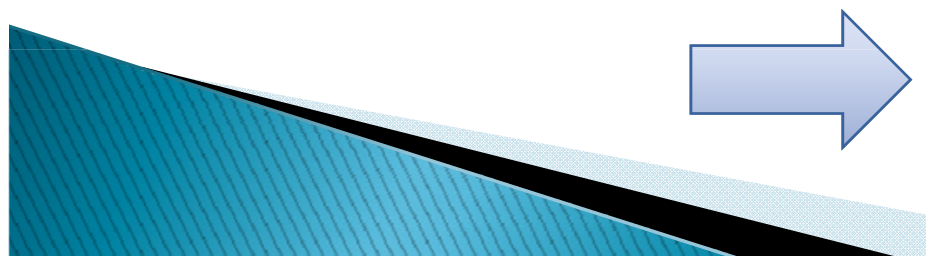

- A monopole ESM requires at least 260 model parameters to achieve accurate predictions. 


\section{Analysis of Results}

\section{$\square$ Results from multipole ESMs}

Overall Prediction Comparison for Case 1

- Regularization does not cause instabilities (more robust).

- The spatially averaged predictions are similar with different multipole orders (accurate up to $5000 \mathrm{~Hz}$ ).

- Multipole ESM requires much fewer number of model parameters than monopole ESM
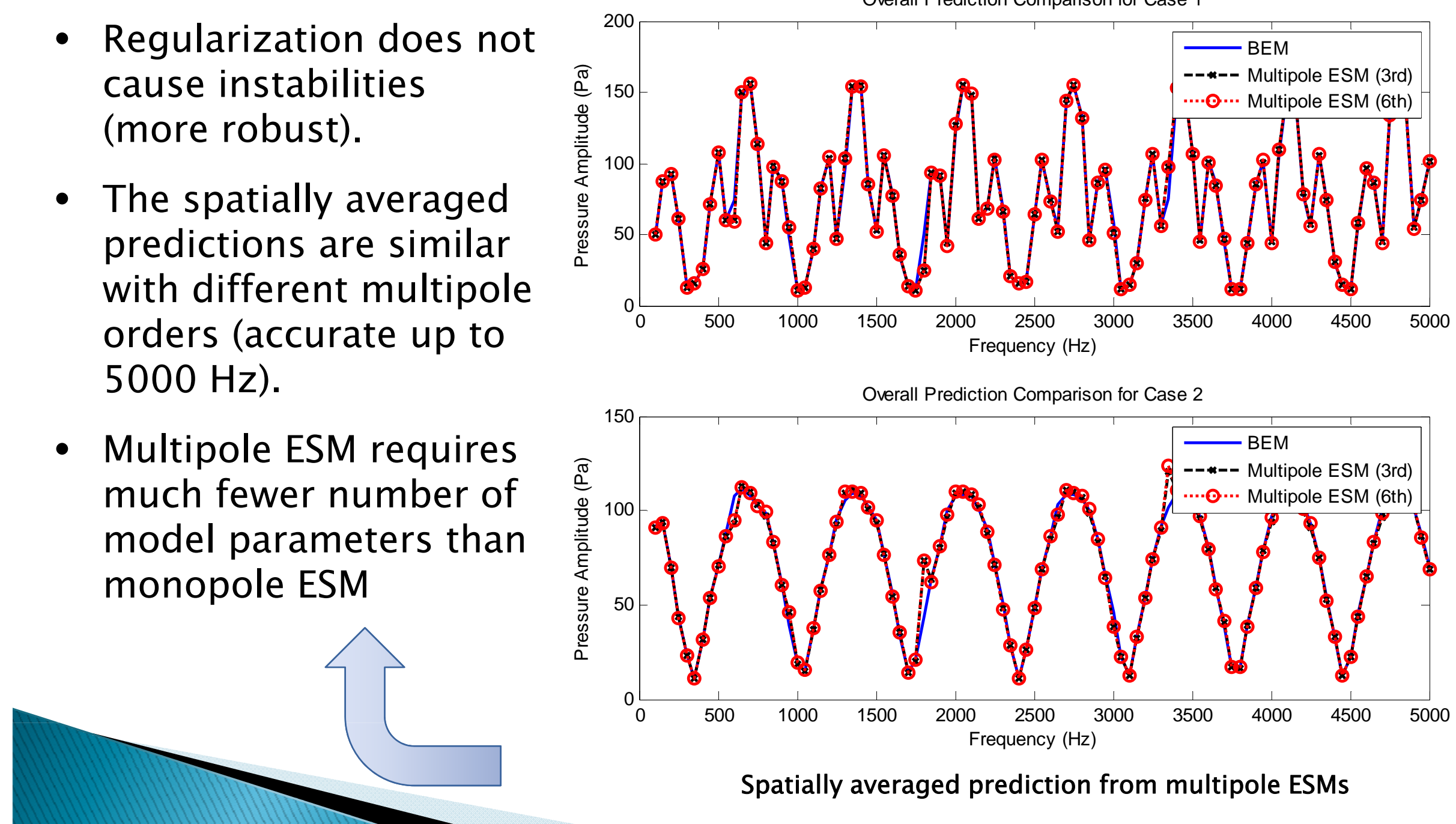

Spatially averaged prediction from multipole ESMs 


\section{Analysis of Results}

$\square$ Results from multipole ESMs
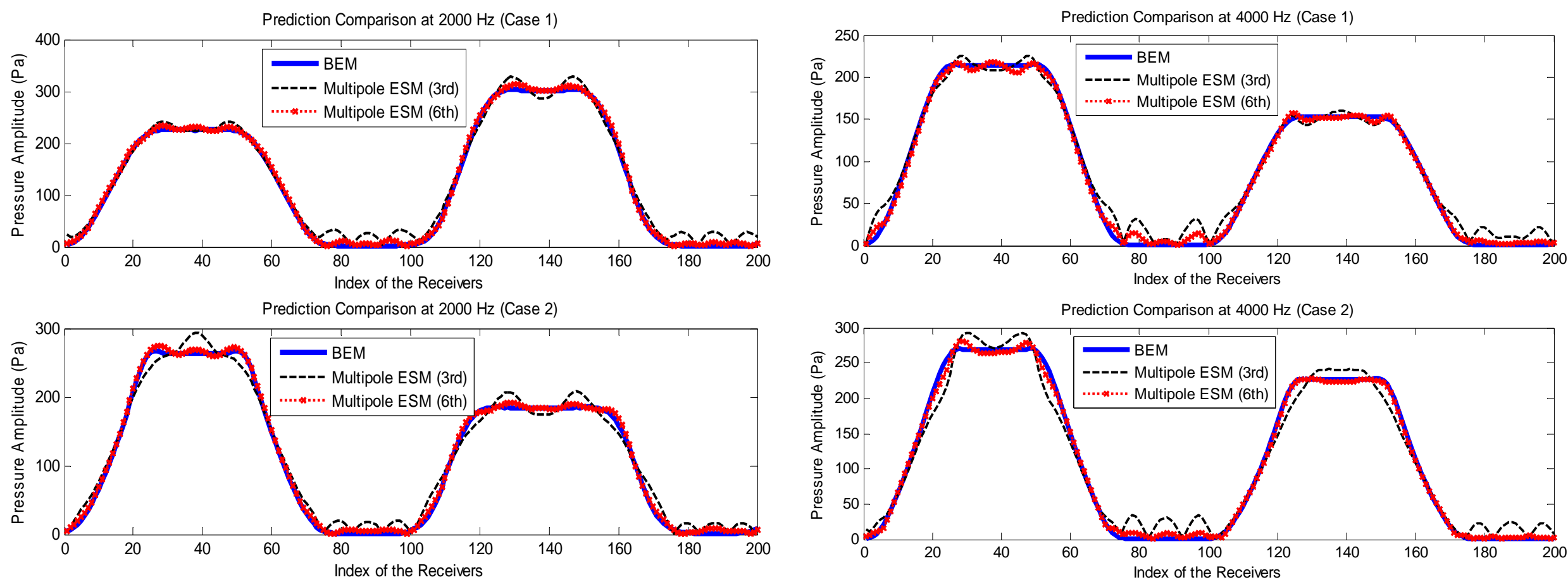

Prediction from multipole ESMs at $2000 \mathrm{~Hz}$

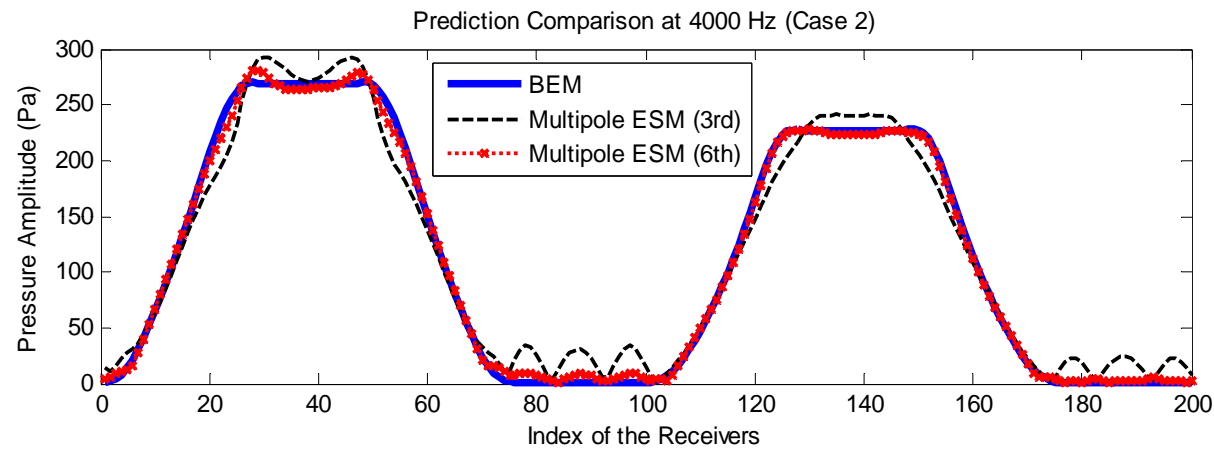

Prediction from multipole ESMs at $4000 \mathrm{~Hz}$

- Increase of multipole order improves the prediction oscillation in space.

- It is flexible to balance the computational effort and the prediction accuracy. (choose an appropriate truncation order 


\section{Conclusions}

$\square$ Equivalent source models are constructed for room acoustics simulations with finite-size source, arbitrary geometry and nonuniform surface normal impedances.

$\square$ In room acoustics ESMs, both outgoing and incoming waves should be included, and the impedance boundary conditions for the room component sound field are used for parameter estimation.

$\square$ Both monopole ESMs and multipole ESMs can achieve accurate performance up to $5000 \mathrm{~Hz}$, but the multipole ESM requires fewer model parameters and is more robust.

$\square$ When using multipole ESM, there is a flexible balance between the computational effort and the prediction accuracy, controlled by choosing an appropriate truncation order.
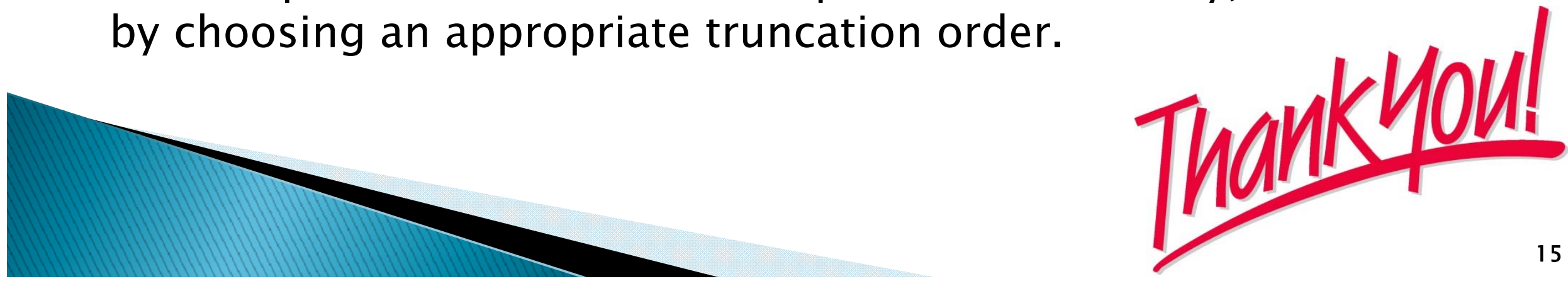